\title{
OVERVIEW OF SUPERCAPACITORS - APPLICATION OF CARBON NANOPARTICLES
}

\author{
Dzhihan Menseidov \\ Institute of Mechanics, Bulgarian Academy of Sciences, \\ Acad. G. Bontchev St., Bl. 4, 1113 Sofia, Bulgaria, \\ University of Telecommunications and Post, \\ 1, Acad. Stefan Mladenov St., 1700 Sofia, Bulgaria, \\ e-mail: menseidov@gmail.com
}

\begin{abstract}
Supercapacitors are ideal electrochemical energy storage devices that predetermine the difference between conventional capacitors and batteries by applying applications to different power and energy requirements. In supercapacitors, as an ordinary capacitor, there are two plates separated by a dielectric and there is an electrolyte inside it, separated plates, and storing more energy than the ordinary capacitor, creating a double layer of distributed charges between two plates made of porous, however, based on carbon materials. In this way, it offers the ability to store or release energy for a few seconds with long cycle life. This study covers a brief overview of the supercapacitor, including the energy storage mechanism, concept, classification, and applications.

Keywords: supercapacitors, energy storage, concept, principle.
\end{abstract}

\section{INTRODUCTION}

The transport sector in developing countries is dependent on oil as an energy source, but today we are witnessing the beginning of a new era with the advent of electric vehicles. This group, of which Bulgaria is a country, imports over $90 \%$ of crude oil, which makes the development of countries economically linked to the price of fuels, which in turn has serious economic and social risks. The gradual decline in oil reserves, together with their escalating prices, and the escalating environmental crisis have made current fossil fuel-based transport unsustainable and undesirable in the long run. Electrification of transport offers an alternative to today's oil-dependent economy. The greater penetration of electric vehicles provides opportunities to shift the

DOI: 10.7546/EngSci.LVIII.21.01.06 
transport demand for energy to the electricity grid and its diverse range of options, which includes Energy Storage Systems (ESS) and a wide choice of generation sources. The production of electricity using numerous and diverse sources such as coal, nuclear energy, solar energy, wind and others offers a reliable and some of them environmentally friendly way to increase energy production capacity. This will be crucial in order to avoid additional stress on the network caused by the load that will be provided by the transport network. Thus, the development of efficient public and private transport systems, including efficient network distribution, is the key to meeting the energy needs of developing countries. There are currently several studies on the integration of the transport network, which are mainly limited to the vehicle to the network (V2G) and pilot projects on the same have been carried out in countries such as Japan, China, USA and the European Union [1].

Electricity is a flexible form of energy and it is not profitable to store it for a long time. So a prominent place is the skillful storage of energy for qualified consumption or utilization. In fact, many developing countries are now facing a boom in the production of renewable energy from the sun, wind and geothermal energy, which are the richest resources. But we must keep in mind the changing nature of solar and wind energy, there is a critical requirement for such energy devices that are safe to operate, cost-effective, have less impact on the environment and can also store energy in excess and they release it when it is in great demand. Such energy storage devices are batteries, fuel cells and supercapacitors will play a key role in our lives. The electrochemical conversion of energy attracted requires our attention, because there are big challenges ahead. The efficiency of the conversion is high and repeated for a large number of cycles. The electric charge works as a clean, efficient and universal oxidizer or reducer for several electrochemical reactions.

\section{ELECTROCHEMICAL SYSTEMS FOR CONVERSION AND STORAGE OF ENERGY}

There is a need to develop energy storage devices that store the collected energy for wide applications. Conventional capacitors, batteries, and fuel cells are commonly used energy storage devices. Batteries and fuel cells are suitable for use in energy storage in industrial and consumer electronic devices due to their high energy density. Batteries can hold large amounts of energy, but charging takes hours. Fuel cells, in turn, have a low energy density. On the other hand, supercapacitors charge instantly but have the capacity to store small amounts of energy. So there is a demand and need for devices that 
can store and release large amounts of electricity very quickly. Therefore, energy storage devices such as electrochemical capacitors are needed, which have a high power density, long cycle life, cost-effective in a compact size. Electrochemical capacitors are also known as supercapacitors due to their extremely high capacitance density.

\section{THEORETICAL BASIS OF A SUPERCAPACITOR}

A Supercapacitor (SC) is a high-capacity electrochemical capacitor with capacitance values much higher than conventional capacitors.

One way to increase the capacitance can be achieved by electrodes with a large area and a very thin dielectric, while electrodes with a small surface area and a denser dielectric are responsible for the lower capacitance. Supercapacitors work in the same way as conventional capacitors. However, supercapacitors can store a large amount of charge, as increased costs can be saved by a large number of pores in high-surface electrodes and charge separation is performed from a reduced distance between the electrolyte and the electrode. The supercapacitor has a standard design and consists of two electrodes, an electrolyte and a separator.

The electrodes are preferably high surface area. The electrolyte is a mixture of positive ions and negative ions dissolved in a conductive liquid. These two electrodes are separated by a separating membrane, which allows the mobility of charged ions. The separator is rolled or folded into a container with a cylindrical shape.

In the case of supercapacitors, the mechanism for storing electric charge is the same, but the charge accumulates at the interface of the conductor and electrolyte. In this way, a double layer is formed due to the accumulated charge. One of the layers is due to the charged electrode, and the other is created due to electrolyte ions. The charge storage process is non-faradic, as no electronic transfer is performed through the electrode-electrolyte interface, and the charge and energy storage is purely electrostatic.

\subsection{Energy storage mechanism}

The energy storage devices considered so far are on the principle of electric two-layer mechanism, in which the charge is stored electrostatically, pseudo capacitive charge storage, in which the costs are stored electrochemically. 


\section{Electrostatic double layer capacity}

Various models have been explained, such as the Helmholtz model, the Grahame model, the Gouy-Chapman model, and the Gouy-Chapman-Stein model, which perform electrodes under potentiostatic control in the electrolyte of supercapacitors.

In general, electrochemical capacitors store charge electrostatically [2]. When the electrode is immersed in the electrolyte, two opposite charges are impulsively formed at the boundary between the electrode and the electrolyte. These charges with different polarity are presented in double electric charging layers, the charging process is schematically illustrated in Fig. 1. On the surface of the electrode is formed a charged layer with a single polarity positive or negative, and in the electrolyte solution is formed a different charged layer with opposite polarity. These two charge layers are separated by a layer of solvent or electrolyte molecules. This layer, separating different charges, acts as a dielectric. These solvate molecules prohibit the flow of charge between the electrode and the electrolyte. When the power supply is properly connected to the electrodes, the two-layer capacitor begins to charge.

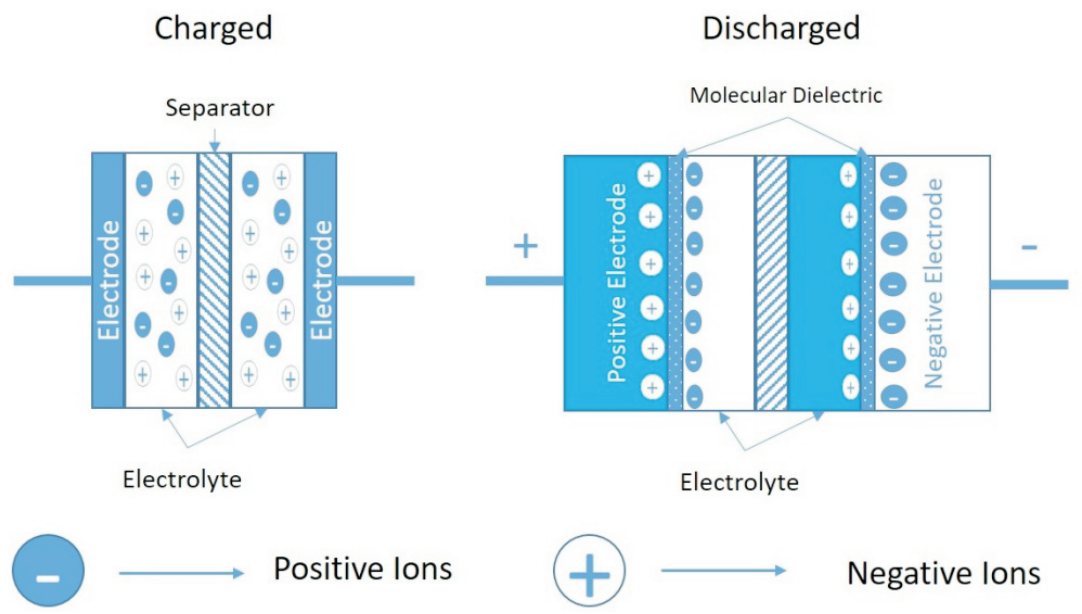

Fig. 1. Schematic drawing of basic double layer principle

When the electrode is immersed in an electrolyte, either the electrolyte ions are adsorbed on the surface of the electrode, or the atoms of the electrode are dissolved in the electrolyte. But each of them develops a separation of charge between the surface of the electrode and the electrolyte. This forms an electrochemical double layer that can be used to store charge. By increasing the applied voltage, the amount of stored charge can be improved. Increasing 
the applied voltage will place excess charges on the surface of the electrode and will repel oppositely charged ions from the electrolyte to line up near the electrode.

High surface and conductivity electrodes are preferred because they outperform the others and are suitable for use in supercapacitors. Carbon materials are characterized by low cost, excellent electrochemical stability, conductivity and excellent machinability making them a preferred material for the manufacture of electrodes [3]. The size of the electrode is a function of the amount of charge stored in per unit voltage in the electrochemical capacitor. The electrostatic storage of energy in the double layers of the capacitors is linear with respect to the stored charge and is equal to the concentration of adsorbed ions. In conventional capacitors the charge is transferred by electrons, it should be noted that in two-layer capacitors the capacitance is related to the limited velocity of the atoms in the electrolyte and the porous resistive structure of the separator. No chemical changes are made in the electrode or electrolyte during the discharge and charge cycles of the two-layer capacitors, which makes the number of these cycles unlimited [4].

\subsection{Classification of supercapacitors}

The properties of supercapacitors are related to the interaction of their materials. In particular, the combination of electrode material and type of electrolyte determines the functionality and thermal and electrical characteristics of the capacitors. A third type has recently been erected that stores the charge electrochemically and electrostatically. Dual layer capacitors use high surface carbon materials such as activated carbon, Carbon Nanofibers (CNFs) and carbon aerogels. Pseudocapacitors use a transition metal oxide or conductive polymer as electrodes. Hybrid capacitors also have asymmetric electrodes, one of which has electrostatic and the other has electrochemical capacity.

\subsection{Materials}

The capacity of the device largely depends on the characteristics of the properties of the electrodes. The electrodes must have properties such as good conductivity, high chemical stability, controlled pore structure, high corrosion resistance, temperature stability, workability, environmental friendliness and low cost. The capacity can be increased due to the ability of the material to perform faradic charge transfers. The thickness of the double layer and the charge stored by the electrochemical mechanism strongly depend on the 
surface of the electrode. Through a porous electrode material with a suitable structure that matches the size of the electrolyte, ions improve the electrochemical characteristics. Appropriate pore size distribution has a greater impact on capacity, specific energy, equivalent series resistance and specific power.

Carbon Nanotubes (CNTs) help to improve the performance of the capacitor due to the highly wettable surface and high conductivity. This makes them a widely used material and carbon nanotubes will be the leading material for the electrodes. The porous nature of CNT allows easy entry of electrolyte ions into the pores of the electrodes. CNTs have a high surface area and a level of precipitation relative to carbon. But the high cost still limits its wide application.

Conductive polymers due to properties such as high electrical conductivity, low cost compared to metal oxides, environmentally friendly, fast charge/discharge, high capacity are considered promising materials for supercapacitors such as electrodes. Some of the tested polymers are polyvinylidene fluoride or polyvinylidene difluoride (PVDF), polyaniline (PAn), polypyrrole (PPY), polythiophene (PTh), and others. Unlike pure carbon, total mass and volume are involved in the storage of the charge for conducting polymers, which leads to a high specific capacity. An important disadvantage of conductive polymers is poor mechanical stability; undergoes cracks, shrinkage, swelling, fracture, etc., which lead to a progressive exaggeration of conductivity [5]. Such electrodes suffer from limited cycling resistance which can be selected by combining them into a nanocomposite with the addition of nanotubes.

\section{CONCLUSION}

To move into the new era of electric transport, supercapacitors must meet the need for a large number of charge or discharge cycles and longer life and where a large amount of energy is required in a relatively short time. The high energy advantage of supercapacitors allows opportunities in power electronics. In the field of uninterruptible power supply, great reliability and security are also needed, where high currents are required for a short time, for starting electric and hybrid vehicles. Due to the excellent performance of the power and the more sparing impact on the environment, supercapacitors are most suitable as starters. At the wind turbine step, the supercapacitors provide backup power, so if the main power fails, then it is also possible to adjust the blade pitch. Supercapacitors can stabilize power in variable load applications such as electric motors, photovoltaic systems, GPS communications, and 
many other areas. Supercapacitors are an integral part of our future and only with the advent of nanocomposites based on conductive polymers and carbon nanoparticles is their rapid development.

\section{ACKNOWLEDGEMENTS}

This work was supported by: The Bulgarian Ministry of Education and Science under the National Research Programme "Young scientists and postdoctoral students" approved by DCM \# 577/17.08.2018.

H2020-SGA-FET-Graphene Flagship-Graphene Core 3.

Marie Skłodowska-Curie Actions (MSCA) Research and Innovation Staff Exchange (RISE) H2020-MSCA-RISE-2016, Project Acronym: Graphene 3DGrant Number: 734164 .

\section{REFERENCES}

[1] Smart Grid around the world, Energy Information Administration (Online), Available: http://www.eia.gov/analysis/studies/electricity/pdf/ intl_sg.pdf.

[2] P. Simon And Y. Gogosti, Materials for electrochemical capacitors, Nature Materials (2008) 7 845-854.

[3] P. Lu, D. Xue, H. Yang, and Y. Liu, Supercapacitor and Nanoscale Research Towards Electrochemical Energy Storage, International Journal of Smart and Nano Materials (2012) First 1-25.

[4] L. L Zhang AND X. S Zhao, Carbon based materials as supercapacitor electrodes, Chem. Soc. Rev. (2009) 38 (9) 2520-2531.

[5] E. Frackowiak, V. Khomenko, K. Jurewicz, K. Lota, and F. Beguin, Supercapacitor based on conducting polymers/nanotubes composites, Journal of Power Sources (2006) 153413.

Received November 17, 2020 